\title{
THEORETICAL ASPECTS OF ASTEROSEISMOLOGY
}

\author{
J. CHRISTENSEN-DALSGAARD \\ Teoretisk Astrofysik Center, Danmarks Grundforskningsfond, and \\ Institut for Fysik og Astronomi, Aarhus Universitet, \\ DK-8000 Aarhus C, Denmark
}

\section{Introduction}

Asteroseismology depends on the ability to determine frequencies for identified modes of stellar oscillation, and to use these frequencies to probe stellar interiors. Thus crucial aspects are the dependence of the frequencies on stellar properties, and the likelihood that modes are excited to observable amplitudes. I provide a brief overview of these issues and some remarks about how the frequencies may be analyzed, as a background for the more detailed presentations elsewhere in the proceedings.

\section{The dependence of frequencies on the properties of a star}

The frequency dependence can in general be separated into the part arising from the spherically averaged stellar structure and the part resulting from effects of rotation, the latter being treated as a small perturbation. The spherical structure determines frequencies that depend on the degree $l$ and radial order $n$ of the modes, but not on their azimuthal order $m$; rotation induces a splitting after $m$.

The dependence of the frequencies of a spherically symmetric star on stellar structure can to a large extent be understood in terms of characteristic frequencies of the star. The most basic of these is the dynamical frequency $\omega_{\mathrm{dyn}}=\left(G M / R^{3}\right)^{1 / 2}$, where $M$ and $R$ are the mass and radius of the star, and $G$ is the gravitational constant; for stellar models related by homology, the pulsation frequencies scale precisely as $\omega_{\text {dyn }}$. Measurement of a single frequency of an identified mode therefore essentially provides information on $\omega_{\text {dyn }}$, i.e., on the mean density of the star; such a measurement is interesting in itself, but hardly constitutes seismology.

The detailed properties of the oscillations depend largely on two characteristic frequencies: the Lamb frequency $S_{l}=[l(l+1)]^{1 / 2} c / r$, where $c$ is the adiabatic sound speed and $r$ is the distance from the centre; and the buoyancy frequency $N$, given by

$$
N^{2}=g\left(\frac{1}{\Gamma_{1}} \frac{\mathrm{d} \ln p}{\mathrm{~d} r}-\frac{\mathrm{d} \ln \rho}{\mathrm{d} r}\right) \simeq g^{2} \frac{\rho}{p}\left(\nabla_{\mathrm{ad}}-\nabla+\nabla_{\mu}\right) \equiv N_{0}^{2}+N_{\mu}^{2},
$$

where $p$ is pressure, $\rho$ is density, $\Gamma_{1}=(\partial \ln p / \partial \ln \rho)_{\text {ad }}$ is the adiabatic exponent, and $g$ is gravity. In the last approximation I assumed the ideal gas law for a fully ionized 
gas. Here $\nabla=\mathrm{d} \ln T / \mathrm{d} \ln p, \nabla_{\text {ad }}$ is its adiabatic value and $\nabla_{\mu}=\mathrm{d} \ln \mu / \mathrm{d} \ln p$, where $\mu$ is the mean molecular weight; also, I introduced

$$
N_{\mu}^{2}=-g \frac{\mathrm{d} \ln \mu}{\mathrm{d} r}=-4 \pi G \rho \frac{\mathrm{d} \ln \mu}{\mathrm{d} \ln m} .
$$

Figure 1. Characteristic frequencies, in units of $\omega_{\text {dyn }}$. Thin lines are for a model of the present Sun and heavy lines for $1.8 M_{\odot}$ models. Long-dashed line: $N$ in the $1.8 M_{\odot}$ ZAMS model; continuous lines: $N$ in evolved $1.8 M_{\odot}$ and solar model; short-dashed lines: $S_{2}$ in evolved $1.8 M_{\odot}$ and solar model. The heavy horizontal line shows the frequency of a mode with contributions both from the p-mode and gmode regions.

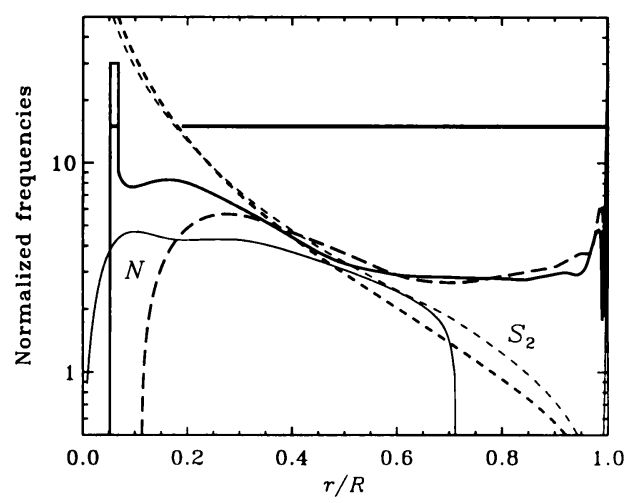

The behaviour of $S_{l}$ and $N$, in units of $\omega_{\text {dyn }}$, is illustrated in Fig. 1 for a model of the present Sun and ZAMS and evolved models of a $1.8 M_{\odot}$ star. It is evident that $S_{l}$ scales approximately homologously, as does $N_{0}$ for the evolving $1.8 M_{\odot}$ model. On the other hand, $N_{\mu}$ reflects the gradient in $\mu$ in evolved models which has resulted from nuclear burning; this is particularly striking in the $1.8 M_{\odot}$ star, where the shrinking convective core has left behind a strong gradient in $\mu$, leading to the peak in $N$.

The local physical nature of a mode is determined by its frequency, relative to $S_{l}$ and $N$ : where $\omega>S_{l}$ and $\omega>N$ the mode behaves as an acoustic wave (or a p mode) whereas where $\omega<S_{l}$ and $\omega<N$ it behaves as a gravity wave (or g mode). The mode is evanescent where the frequency is between $S_{l}$ and $N$, growing or decaying exponentially with $r$. As illustrated in Fig. 1, modes generally have p-mode behaviour at high frequency and g-mode behaviour at low frequency; however, a given mode can have different characters in different parts of the star. The global nature of the mode is then essentially determined by the region where the local energy is largest.

For high-frequency modes in solar-like stars, including the five-minute oscillations of the Sun, $|\omega / N| \gg 1$. Such modes behave essentially as standing sound waves, and the frequency is determined by the sound speed. For low-degree modes (of main relevance for distant stars) the cyclic frequency of a mode of degree $l$ and radial order $n$. satisfies the asymptotic relation $\nu_{n l} \simeq \Delta \nu(n+l / 2+\alpha)+\epsilon_{n l}$ (e.g. Tassoul 1980). Here $\Delta \nu=\left(2 \int_{0}^{R} \mathrm{~d} r / c\right)^{-1}$ is the inverse of the sound travel time across a stellar diameter; this can be estimated as the large frequency separation $\Delta \nu_{n l} \simeq \nu_{n l}-\nu_{n, l-1}$. The correction term $\epsilon_{n l}$ leads to a small frequency separation $\delta \nu_{n l}=\nu_{n l}-\nu_{n-1 l+2}$, which is largely determined by the sound-speed gradient in the core of the star. Since for an ideal gas $c^{2} \simeq \Gamma_{1} k_{\mathrm{B}} T / \mu m_{\mathrm{u}}$ ( $k_{\mathrm{B}}$ being Boltzmann's constant and $m_{\mathrm{u}}$ the atomic mass unit), $\delta \nu_{n l}$ is sensitive to the composition structure of the star and hence its evolutionary state. As a result of this sensitivity, $\delta \nu_{n l}$ does not scale as $\omega_{\text {dyn }}$ with stellar mass and radius, while $\Delta \nu_{n l}$ essentially scales homologously. 
The departures from the simple asymptotic expression contain potentially important information. In particular, sharp features in the stellar structure may induce oscillations in the frequencies, as functions of mode order. An important example is the localized decrease in $\Gamma_{1}$ associated with the second helium ionization zone; the resulting perturbation in the frequencies has been used to infer the solar helium abundance (e.g. Vorontsov et al. 1991; Kosovichev et al. 1992; Pérez Hernández \& ChristensenDalsgaard 1994), and corresponding information may become available from stellar observations (Lopez et al. 1997; Pérez Hernández \& Christensen-Dalsgaard 1997). A second example is provided by the oscillations induced by the possible sharp variation in the sound-speed gradient at the edges of convective regions, particularly in the presence of overshoot. Analysis of this feature in the solar case has placed limits on the extent of overshoot beneath the solar convection zone (e.g. Basu et al. 1994; Monteiro et al. 1994; see also Monteiro et al., these proceedings).

Figure 2. Modes of degree $l=2$ in an evolution sequence of $1.8 M_{\odot}$ models, indicated by their effective temperature $T_{\text {eff }}$. The frequencies $\sigma$ are given in units of $\sqrt{3} \omega_{\text {dyn }}$. Filled circles indicate unstable modes, open circles stable modes. (From Goupil et al. 1996.)

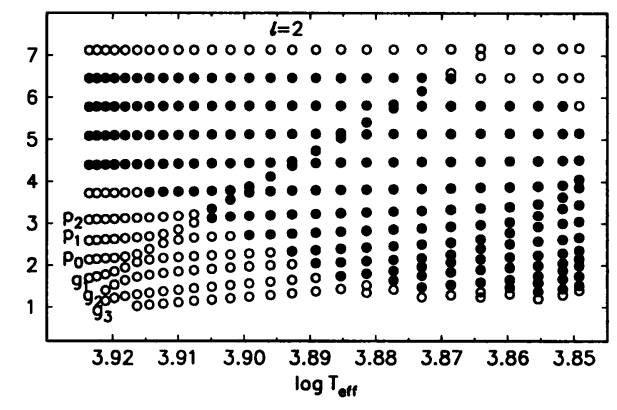

The approximately uniform spacing of the solar-like oscillation spectrum substantially simplifies analysis of of observed modes of oscillations. In other types of pulsating stars, the spectrum of oscillations can be considerably more complex, with a corresponding greater richness in the potentially available information. This is the case for evolved stars with a (possibly earlier) convective core, where the steep maximum in the buoyancy frequency (cf. Fig. 1) allows for g-mode like behaviour at frequencies otherwise characteristic for p modes. As the star evolves, $S_{l}$ and $N_{0}$ scale as $\omega_{\text {dyn }}$, i.e., as $R^{-3 / 2}$. On the other hand, $N_{\mu}$ increases with the increasing composition gradient and central density as the star evolves. It follows that for modes behaving like $\mathrm{p}$ modes the frequencies decrease with evolution while $\mathrm{g}$ modes trapped in the region of composition gradient have increasing frequencies. This is illustrated in Fig. 2 for a $1.8 M_{\odot}$ evolution sequence. Since frequencies in units of $\omega_{\text {dyn }}$ are shown, the p-mode values are essentially constant, while the g-mode frequencies reflect the increasing $N_{\mu}$. Where the frequencies of two modes of different nature approach, the modes undergo avoided crossing, where the modes exchange physical nature. Near the avoided crossing the two modes have a mixed character, with substantial amplitude in both the regions of p-mode and g-mode behaviour. Evidently the observational identification of such a pair of modes would provide important information about the properties of the edge of the convective core, possibly including evidence for convective overshoot.

Rotation (or other departures from spherical symmetry) induces a dependence of the frequencies on the azimuthal order $m$ of the mode. For slow rotation, one may neglect quadratic effects in the angular velocity $\Omega$, including the distortion of the equilibrium structure. Then the effect of rotation on the frequencies is solely a lifting 
of the degeneracy of the frequencies in $m$. If $\Omega$ is assumed to depend only on $r$

$$
\omega_{n l m}=\omega_{n l 0}+m \beta_{n l} \int_{0}^{R} K_{n l}(r) \Omega(r) \mathrm{d} r
$$

(e.g. Gough 1981). Here the kernels $K_{n l}(r)$ have been defined such as to have unit integral. For high-order $\mathrm{p}$ modes $\beta_{n l}$ is close to one, and the rotational splitting $\left(\omega_{n l m}-\omega_{n l 0}\right) / m$ is approximately an average of $\Omega$ weighted by the energy density in the mode. For high-order g modes, on the other hand, $\beta_{n l} \simeq 1-[l(l+1)]^{-1}$. The pattern of approximately uniformly split frequencies indicated by Eq. (3) simplifies the mode identification, although it must be kept in mind that not all the components for a given multiplet $n l$ need be excited, or visible.

With more rapid rotation, second-order effects of rotation must be taken into account. These change the equilibrium structure of the star, leading to frequency changes for all modes; also, they cause an additional contribution to the frequency splitting which, for uniform rotation and $l \gtrsim 2$ can be approximated by

$$
\delta \omega_{n l m}^{(2)} \simeq-f_{n l} \frac{\Omega^{2} R^{3}}{G M} \omega_{n l 0} P_{2}\left(\frac{m}{l+1 / 2}\right),
$$

where $P_{2}(x)=\left(3 x^{2}-1\right) / 2$ is a Legendre polynomial (e.g. Gough \& Thompson 1990; see also Dziembowski \& Goode 1992). The factor $f_{n l}$ is approximately $1 / 3$ for highorder or high-degree p modes; thus the magnitude of the quadratic effect in $\Omega$ corresponds approximately to the surface ratio between the centrifugal force and gravity. Notice that this relation results in a non-uniform spacing of the split frequencies. For rapidly rotating stars, including many $\delta$ Scuti stars, the splitting is comparable with the separation between the multiplet frequencies $\omega_{n l}$ of the corresponding non-rotating star, leading to very complex spectra of oscillations.

\section{Mode excitation and selection}

Stellar modes of oscillation are of little interest unless excited to observable amplitudes. Two distinct types of excitation are relevant. In the Sun, and presumably other solar-like stars, the oscillations are intrinsically stable. Here the modes arise through stochastic driving by the turbulent convection (see also Nordlund \& Stein, these proceedings). The resulting amplitudes are determined by the balance between the linear damping rate of the modes (in itself strongly affected by the perturbation in the turbulent flux and pressure) and the energy input from the turbulence, at the frequency of oscillation. This in turn depends on the spectrum of turbulence and the properties of the oscillation eigenfunctions in the region, very near the surface, of nearly sonic convection. In the Sun, these factors combine to provide driving to substantial amplitudes in a fairly broad frequency region, between around 2 and $5 \mathrm{mHz}$; the resulting amplitudes are largely independent of the degree of the mode, since the scale of the relevant convection is much smaller than the scale of the oscillations.

As a rough guide the energy in a stochastically excited mode corresponds to the energy of a convective eddy with the corresponding time scale (e.g. Goldreich \& Keeley 1977); this was applied by Christensen-Dalsgaard \& Frandsen (1983) to estimates of the expected amplitudes of solar-like oscillations in other stars. From the results Kjeldsen \& Bedding (1995) found an approximately linear dependence of the amplitude on the ratio $L / M$ between the luminosity and mass of the star. 
Houdek (1996; see also Houdek et al. 1997) carried out an extensive survey of the stability of oscillations in main-sequence stars and the expected amplitudes of stochastically excited modes; he used the non-local mixing-length theory of Gough (1976) and Balmforth (1992). The predicted amplitudes were fairly similar to those obtained by Christensen-Dalsgaard \& Frandsen (1983).

The second type of excitation occurs where the modes are overstable. In this case the oscillation extracts mechanical energy from the flux of energy through the star, operating as a heat engine with matter being heated at compression and cooled at expansion. The contributions to the driving typically arise from specific regions in the star and must compete with damping elsewhere. The general mechanism was first proposed by Eddington (1926) and elaborated to explain the excitation of oscillations in Cepheids by Zhevakin (1953) and Cox \& Whitney (1958). It involves a perturbation to opacity such that energy is preferentially trapped at compression. It is typically associated with a local bump in the opacity, caused by the ionization of a specific element. To result in net driving, the bump must be located at the transition between nearly adiabatic and strongly nonadiabatic oscillation (see also the discussion by Cox 1974): this transition occurs at the location $m_{\mathrm{t}}$ in mass such that the thermal time scale $\tau_{\text {th }}\left(m_{\mathrm{t}}\right)$ of matter outside $m_{\mathrm{t}}$ is equal to the oscillation period, i.e.,

$$
\omega \tau_{\mathrm{th}}\left(m_{\mathrm{t}}\right) \simeq \omega L^{-1} \int_{m_{\mathrm{t}}}^{M} c_{p} T \mathrm{~d} m \sim 1,
$$

where $c_{p}$ is the specific heat at constant pressure. Thus the condition for instability is that $m_{\mathrm{t}} \simeq m_{\mathrm{b}}$, the mass corresponding to the relevant bump in the opacity.

Instability of Cepheids and $\delta$ Scuti stars arises from the opacity feature at the second ionization of helium. It was shown by Cox \& Whitney that this accounted for the location of the Cepheid instability strip. The recent revisions of opacity tables (e.g. Rogers \& Iglesias 1992; Seaton et al. 1994) have shown a bump at higher temperature, arising predominantly from bound-bound transitions in iron-group elements, which gives rise to the instability of the $\beta$ Cephei stars and other B stars (e.g. Cox et al. 1992; Moskalik \& Dziembowski 1992). Interestingly, in the recently discovered hot pulsating subdwarf stars, the EC 14026 stars (Kilkenny et al. 1997; see also Stobie et al., these proceedings) radiative levitation appears to concentrate iron in the critical region, enhancing the instability (Charpinet et al. 1997; Fontaine, these proceedings).

It follows from Eq. (5) that the location of the transition depends on the frequency of oscillation: with increasing frequency, $\tau_{\mathrm{th}}\left(m_{\mathrm{t}}\right)$ must decrease, i.e., the transition move closer to the surface. With increasing effective temperature the relevant bumps in the opacity move closer to the surface; thus instability may be expected at higher frequency. Hence, higher-order acoustic modes are excited to the blue side of the instability strip corresponding to the fundamental radial oscillation. Conversely, on the cool side of the instability strip modes of lower frequency, i.e., g modes, may be excited. These predictions are generally confirmed by detailed stability calculations. In particular, the slowly pulsating B stars (e.g. Aerts et al., these proceedings) appear to pulsate in $\mathrm{g}$ modes, excited by the opacity mechanism operating on the iron bump in the opacity (Dziembowski et al. 1993). Similarly, it is tempting to identify the recently discovered $\gamma$ Doradus stars, at lower $T_{\text {eff }}$ than the $\delta$ Scuti stars (e.g. Balona et al. 1994) as caused by excitation of $g$ modes by the second helium ionization bump; here, however, the understanding of the excitation is complicated by effects of convection.

For overstable modes, a separate mechanism is needed to explain the final limiting amplitude and hence the selection of observable modes. The amplitude-limiting 
mechanism for stars near the main sequence is so far highly uncertain. They generally pulsate in several modes (thus making them more interesting from a seismological point of view), with amplitudes too small to saturate the driving mechanism. It has been proposed that the amplitudes are limited by resonant interactions involving stable modes (e.g. Dziembowski \& Królikowska 1985; Dziembowski et al. 1988), although no detailed predictions of spectra for specific stars have been made so far; even so, this might possibly explain the often complex spectra observed. It is interesting, however, that increasing observational sensitivity appears to reveal steadily more modes, as has been shown dramatically by the observations of FG Virginis (Breger et al. 1995, 1997; see also Breger, these proceedings).

\section{Analysis of observed frequencies}

A prerequisite for using the observed frequencies to obtain a test of the properties of the stellar interior is the identification of the observed modes. For solar-like oscillations this may be possible from the regular nature of the expected spectrum ( $c f$. Section 2). Indeed, Christensen-Dalsgaard \& Gough (1980) on this basis obtained an identification, confirmed by later observations, of the solar low-degree modes. Furthermore, the data can be analysed in terms of the large and small frequency separations $\Delta \nu_{n l}$ and $\delta \nu_{n l}$ which may be compared with models, even without a precise assignment of $n$. This provides a measure of the mass and, because of the dependence of $\delta \nu_{n l}$ on the core structure, of the age of the star (e.g. Ulrich 1986; Christensen-Dalsgaard 1988). In fact, the values of $\Delta \nu_{n l}$ and $\delta \nu_{n l}$ inferred from observations of $\eta$ Boo (Kjeldsen et al. 1995) were in good agreement with evolution models of the star (ChristensenDalsgaard et al. 1995; Guenther \& Demarque 1996). However, it should be noted that the determination of mass and age from the frequency separations presupposes (unrealistically) that all other parameters of the star are known (e.g. Gough 1987).

Given the uncertainty in basic stellar parameters, much can be gained by combining the observed frequencies with other, more 'classical' observations of stellar properties. This is particularly true for systems of two or more stars which may be assumed to share certain properties, such as age and chemical composition. Brown et al. (1994) developed a method for combining such data for binary systems and demonstrated the potential improvements, resulting from access to frequency data, in the determination of the parameters of the system. A preliminary application of this technique to models of stellar clusters was carried out by Audard et al. (1997).

The identification of solar-like modes is simplified by the broad-band nature of their excitation. In $\delta$ Scuti stars it appears that the more selective excitation and amplitude limitation mechanisms lead to complex spectra, which are difficult to interpret. Viskum et al. (1997) demonstrated how the degrees of the modes could be identified by combining observations in Balmer-line equivalent width and broad-band intensity (see also Breger, these proceedings). As a result, reasonably reliable identifications are available for a substantial number of modes in the very mode-rich star FG Vir. Similar techniques have been applied previously, on the basis of amplitude ratios and phase differences between different types of observations (e.g. Watson 1988).

Although a problem for the mode identification, the variety of modes in $\delta$ Scuti stars is a great bonus for investigations of the stellar interiors. Here I consider the possibility for carrying out rotational inversion, as presented by Goupil et al. (1996). They noted that there was a fairly broad range of unstable modes in the somewhat evolved $1.8 M_{\odot}$ star which they investigated, including several g modes trapped at 

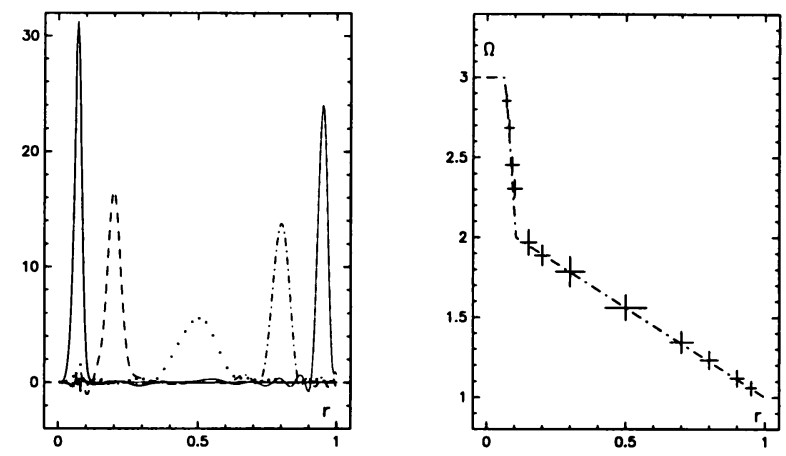

Figure 3. The left panel shows averaging kernels obtained from inversion of modes in an evolved $1.8 M_{\odot}$ model. The right panel shows the assumed rotation profile (dot-dashed line) and the rotation rates inferred from the inversion. The abscissa is fractional radius. (From Goupil et al. 1996.)

the edge of the core ( $c f$. Fig. 2). They argued that modes of degree up to 6 might be visible in observations sensitive to very low-amplitude oscillations and considered the corresponding kernels for the first-order rotational splitting ( $c f$. Eq. 3). These were sufficiently varied to allow inversion for the internal rotation rate throughout the star, with fairly high resolution in the core and near the surface. To demonstrate this, Goupil et al. carried out a SOLA inversion ( $c f$. Thompson, these proceedings), illustrated in Fig. 3, for an artificial rotation profile. The averaging kernels (left panel) provide a measure of the resolution of the inversion; the high resolution in the core reflects the availability of $g$ modes peaked in this region. As shown by the right panel, the inversion does in fact successfully recover the assumed rotation law.

It is fair to say that current observations are not adequate for obtaining the required data, particularly at relatively high degree. However, there is hope that future space experiments, such as COROT (e.g. Catala et al. 1995) may reach the required sensitivity and frequency resolution. In any case, the analysis demonstrates the potential for investigating stellar interiors when data on $g$ modes are available.

Acknowledgements. I am grateful to Goupil et al. for providing Figs 2 and 3, and to M. J. Thompson for useful discussions. This work was supported in part by the Danish National Research Foundation through the establishment of the Theoretical Astrophysics Center.

\section{References}

Audard, N., Brown, T. M., Christensen-Dalsgaard, J. \& Frandsen, S., 1997. In Poster Volume; Proc. IAU Symp. 181: Sounding Solar and Stellar Interiors, eds Schmider, F.X. \& Provost, J., Nice Observatory, in press

Balmforth, N. J., 1992. Mon. Not. R. astr. Soc., 255, 603

Balona, L. A., Krisciunas, K. \& Cousins, A. W. J., 1994. Mon. Not. R. astr. Soc., 271, 905

Basu, S., Antia, H. M. \& Narasimha, D., 1994. Mon. Not. R. astr. Soc., 267, 209

Breger, M., Handler, G., Nather, R. E., et al., 1995. Astron. Astrophys., 297, 473

Breger, M., Zima, W., Handler, G., et al., 1997. Delta Scuti Star Newsletter, No. 11, 21 
Brown, T. M., Christensen-Dalsgaard, J., Mihalas, B. \& Gilliland, R. L., 1994. Astrophys. $J ., 427,1013$

Catala, C., Auvergne, M., Baglin, A., et al., 1995. In Proc. Fourth SOHO Workshop: Helioseismology, eds Hoeksema, J. T., et al., ESA SP-376, ESTEC, Noordwijk, p. 549

Charpinet, S., Fontaine, G., Brassard, P., Chayer, P., Rogers, F., Iglesias, C. A \& Dorman, B., 1997. Astrophys. J., 483, L123

Christensen-Dalsgaard, J., 1988. In Proc. IAU Symp. 123, Advances in helio- and asteroseismology, eds Christensen-Dalsgaard, J. \& Frandsen, S., Reidel, Dordrecht, p. 295

Christensen-Dalsgaard, J. \& Frandsen, S., 1983. Solar Phys., 82, 469

Christensen-Dalsgaard, J. \& Gough, D. O., 1980. Nature, 288, 544

Christensen-Dalsgaard, J., Bedding, T. R. \& Kjeldsen, H., 1995. Astrophys. J., 443, L29

Cox, A. N., Morgan, S. M., Rogers, F. J. \& Iglesias, C. A., 1992. Astrophys. J., 393, 272

Cox, J. P., 1974. Rep. Prog. Phys., 37, 563

Cox, J. P. \& Whitney, C., 1958. Astrophys. J., 127, 561

Dziembowski, W. A. \& Goode, P. R., 1992. Astrophys. J., 394, 670

Dziembowski, W. A., Moskalik, P. \& Pamyatnykh, A. A., 1993. Mon. Not. R. astr. Soc., $\mathbf{2 6 5}, 588$

Dziembowski, W. \& Królikowska, M., 1985. Acta Astron., 35, 5

Dziembowski, W., Królikowska, M. \& Kosovichev, A., 1988. Acta. Astron., 38, 61

Eddington, A. S., 1926. The Internal Constitution of the Stars, Cambridge University Press

Goldreich, P. \& Keeley, D. A., 1977. Astrophys. J., 212, 243

Gough, D. O., 1976. In Problems of stellar convection, IAU Colloq. No. 38, Lecture Notes in Physics, vol. 71. eds Spiegel, E. \& Zahn, J.-P., Springer-Verlag, Berlin, p. 15

Gough, D. O., 1981. Mon. Not. R. astr. Soc., 196, 731

Gough, D. O., 1987. Nature, 326, 257

Gough, D. O. \& Thompson, M. J., 1990. Mon. Not. R. astr. Soc., 242, 25

Goupil, M.-J., Dziembowski, W. A., Goode, P. R. \& Michel, E., 1996. Astron. Astrophys., 305, 487

Guenther, D. B. \& Demarque, P., 1996. Astrophys. J., 456, 798

Houdek, G., 1996. Pulsation of solar-type stars, PhD Dissertation, University of Vienna.

Houdek, G., Gough, D. O., Balmforth, N. J. \& Christensen-Dalsgaard, J., 1997. To be submitted to Astron. Astrophys.

Kilkenny, D., Koen, C., O’Donoghue, D. \& Stobie, R. S., 1997. Mon. Not. R. astr. Soc., 285, 640

Kjeldsen, H. \& Bedding, T. R., 1995. Astron. Astrophys., 293, 87

Kjeldsen, H., Bedding, T. R., Viskum, M. \& Frandsen, S., 1995. Astron. J., 109, 1313

Kosovichev, A. G., Christensen-Dalsgaard, J., Däppen, W., Dziembowski, W. A., Gough, D. O. \& Thompson, M. J., 1992. Mon. Not. R. astr. Soc., 259, 536

Lopes, I., Turck-Chièze, S., Michel, E. \& Goupil, M.-J., 1997. Astrophys. J., 480, 794

Monteiro, M. J. P. F. G., Christensen-Dalsgaard, J. \& Thompson, M. J., 1994. Astron. Astrophys., 283, 247

Moskalik, P. \& Dziembowski, W. A., 1992. Astron. Astrophys., 256, L5

Pérez Hernández, F. \& Christensen-Dalsgaard, J., 1994. Mon. Not. R. astr. Soc., 269, 475

Pérez Hernández, F. \& Christensen-Dalsgaard, J., 1997. Mon. Not. R. astr. Soc., in press

Rogers, F. J. \& Iglesias, C. A., 1992. Astrophys. J. Suppl., 79, 507

Seaton, M. J., Yan, Y., Mihalas, D. \& Pradhan, A. K., 1994. Mon. Not. R. astr. Soc., 266, 805

Tassoul, M., 1980. Astrophys. J. Suppl., 43, 469

Ulrich, R. K., 1986. Astrophys. J., 306, L37

Viskum, M., Dall, T. H., Bruntt, H., et al., 1997. In A half century of stellar pulsation interpretations: A tribute to Arthur N. Cox, eds Guzik, J. A. \& Bradley, P., ASP Conf. Ser., in press

Vorontsov, S. V., Baturin, V. A. \& Pamyatnykh, A. A., 1991. Nature, 349, 49

Watson, R. D., 1988. Astrophys. Space Sci., 140, 255

Zhevakin, S. A., 1953. Astron. Zh., 30, 161 